\title{
The Genetics of Obesity
}

\author{
Blanca M. Herrera • Cecilia M. Lindgren
}

Published online: 8 October 2010

(C) The Author(s) 2010. This article is published with open access at Springerlink.com

\begin{abstract}
Obesity is a result of excess body fat accumulation. This excess is associated with adverse health effects such as CVD, type 2 diabetes, and cancer. The development of obesity has an evident environmental contribution, but as shown by heritability estimates of $40 \%$ to $70 \%$, a genetic susceptibility component is also needed. Progress in understanding the etiology has been slow, with findings largely restricted to monogenic, severe forms of obesity. However, technological and analytical advances have enabled detection of more than 20 obesity susceptibility loci. These contain genes suggested to be involved in the regulation of food intake through action in the central nervous system as well as in adipocyte function. These results provide plausible biological pathways that may, in the future, be targeted as part of treatment or prevention strategies. Although the proportion of heritability explained by these genes is small, their detection heralds a new phase in understanding the etiology of common obesity.
\end{abstract}

Keywords BMI · Common obesity · Genetics · GWA · Susceptibility. WHR

\section{Introduction}

Obesity results from a chronic surplus of energy intake compared to energy expenditure, which leads to storage of excessive amounts of triglycerides in adipose tissue [1].

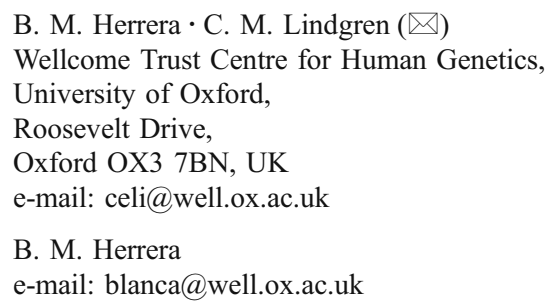

The adverse metabolic effects caused by obesity may result in increased risk of type 2 diabetes, many forms of cancer, fatty liver disease, hormonal disturbances, hypertension, cardiovascular disease (CVD) and increased mortality [25]. Body mass index (BMI) is commonly used as a surrogate measure of overall obesity and, accordingly the World Health Organization, classifies a person with a $\mathrm{BMI} \geq 30 \mathrm{~kg} / \mathrm{m}^{2}$ as obese and a $\mathrm{BMI} \geq 40 \mathrm{~kg} / \mathrm{m}^{2}$ as extremely obese [6].

In the United States, the current prevalence of obesity among adults is about $33 \%$ [7], compared to estimates four decades earlier showing a prevalence of about $13 \%$. Similarly, in the United Kingdom, prevalence has increased to about $23 \%$ [8] and these prevalence figures are reflected throughout the rest of the world [9-12]. Current estimates suggest that by 2015 more than 700 million individuals worldwide will be obese [6]. Prevalence is likely increasing as a result of changes in lifestyle, decreased physical activity, and socioeconomic development, among others. An important concern is that obesity rates are also increasing in children and adolescents all over the world $[6,7]$, predisposing them to poor health from an early age. Obesity is now recognized as an epidemic [6], and despite current intensive efforts to reduce obesity by diet, exercise, education, surgery, and drug therapies, an effective, longterm solution to this epidemic is yet to be provided.

\section{The Genetic Etiology of Obesity}

Obesity is commonly classified into subgroups depending on suspected etiology: monogenic obesity (extremely severe obesity in the absence of developmental delays), syndromic obesity (clinically obese subjects additionally distinguished by mental retardation, dysmorphic features, and organ-specific developmental abnormalities), and poly- 
genic or common obesity, which affects the general population (but may have associated health risks, such as increased risk of CVD).

The first single gene defect causing monogenic obesity was described in 1997, and to date, there are about 20 single gene disruptions that result in an autosomal form of obesity [1]. Interestingly, all these mutations position the leptin/melanocortin pathway in the central nervous system (CNS) as critical in the regulation of whole-body energy homeostasis [13], and obesity in these cases appears to be the result of increased appetite and diminished satiety. Syndromic obesity arises from discrete genetic defects or chromosomal abnormalities at several genes, and can be autosomal or X-linked. One of the most well-known forms of syndromic obesity is Prader-Willi syndrome (PWS), which is caused by a chromosomal abnormality of an imprinted region on chromosome 15q11-q12. PWS is characterized by early-onset obesity resulting from hyperphagia caused by CNS dysfunction [14]. Because both monogenic and syndromic forms of obesity tend to have high penetrance, detection of causal genetic variants has been quite fruitful [15]. The remainder of this review will focus on the genetics of common forms of obesity.

It is worth noting that sex and age are associated with differences in obesity and body composition. For instance, women tend to store more fat subcutaneously rather than in visceral adipose tissue, so at the same BMI, women will tend to carry more body fat than men [7]. Fat distribution follows two general patterns: android (adipose deposition in the abdominal area) and gynoid (adipose deposition around the hips). Android fat distribution is an established, independent risk factor for CVD and type 2 diabetes [16], whereas the gynoid pattern is thought to be protective or inversely correlated [16]. To account for these differences in fat distribution, waist-to-hip ratio $(\mathrm{WHR}=$ waist circumference $[\mathrm{WC}] /$ hip circumference) is commonly used and BMI and WHR are correlated $\left(r^{2} \sim 0.6\right)[17 \bullet \bullet]$.

\section{The Epidemiology and Heritability of Common Obesity}

Epidemiologic studies of common obesity have shown that concordance for obesity decreases in parallel with the degree of relatedness, pointing to a genetic component in obesity susceptibility. For instance, the concordance rate between monozygotic twin pairs is more than twice that of dizygotic pairs $(\sim 0.68$ vs $\sim 0.28)$ [18-21]. In comparison, in adoptee studies, the BMI of the adopted individual is correlated closest with the biological parents' BMI, further highlighting the role of the genetic factors over the shared familial environment $[22,23]$. The high heritability $\left(h^{2}\right)$ for different measures of obesity-BMI $\left(h^{2}=0.4-0.7\right)$ [24], subscapular skinfold thickness $\left(h^{2} \sim 0.77\right)$ [25], WC $\left(h^{2} \sim\right.$
0.76) [21, 26], and WHR $\left(h^{2} \sim 0.45\right)$ [26] -highlight the effect of genetics in increasing risk to obesity. Nevertheless, exposure to an obesogenic environment is necessary for the development of obesity. One hypothesis is that genes that once provided an evolutionary advantage (by allowing maximum efficiency of nutrient storage) are severely challenged when exposed to obesogenic environments [27], although this remains to be formally tested and proved.

\section{Finding Genes for Common Obesity}

The search for obesity susceptibility variants was initially carried out using candidate-gene association studies or linkage analysis. Although these were exceptional tools in the discovery of genes for syndromic and monogenic obesity [15], the success of finding genes for common obesity susceptibility was limited, with very few reproducible results [15]. Association studies (basically comparing allele frequencies between cases and controls) were affected by lack of power in small size study cohorts, limited knowledge of the biology and physiology affecting the candidate gene selection, heterogeneity in the samples, poor phenotyping, and the high cost and effort of genotyping. Numerous linkage studies using affected, related individuals were carried out using microsatellite markers to identify regions of linkage to obesity [15]. However, despite intense efforts, no linkage regions were unambiguously replicated, even after meta-analysis of 37 studies (including 31,000 individuals) [28], demonstrating the limitations of the linkage approach.

\section{Insights into the Genetics of Common Obesity}

The first substantial advances in the discovery of obesity susceptibility loci were made in 2007 [29••, 30]. This was possible through technical and analytical developments allowing for genome-wide association studies (GWAS). GWAS capitalize on the realization that common genetic markers can be inherited together as "blocks" due to linkage disequilibrium [31]. This allowed investigators to capture about $80 \%$ of all common variations ( $>14$ million variants [32]) using as few as 500,000 carefully chosen single nucleotide polymorphisms (SNPs) [33, 34]. Genotyping such selected sets of markers is an attractive approach that allows the capture of most common variation in a single array. The initial success of finding the first robustly associated obesity susceptibility locus, FTO (fat mass and obesity associated), in 2007, [29••, 30, 35••-38$40 \cdot \bullet$ revealed the small effects sizes of obesity genes and propagated the notion that increasing power and sample size of studies was necessary to identify further suscepti- 
bility loci. As a result, even larger and better powered studies have followed, as have multicenter collaborative studies and meta-analyses (Table 1), which have accumulated more than 20 replicated obesity loci (Fig. 1).

Importantly, in most cases we do not know which gene(s) in these loci are contributing to obesity, and the identity of the causative variants is currently unknown. However, in the majority of cases, we can identify the most physiologically likely candidate-gene within/near the associated SNP and further research is needed to verify whether these are the obesity-associated ones or not. Overall, many genes within the associated regions have been reported to fall within two broad categories: genes affecting CNS function and those that are suggested to operate peripherally, often through adipose tissue.

Several of the genes located within or near the associated regions are highly expressed in the CNS, particularly in the hypothalamus [36・0], and appear to be involved in appetite, satiety, energy expenditure, and behavior. This suggests that similar mechanisms would be affected in common forms as in monogenic forms of obesity. FTO has a potential role in nucleic acid demethylation and is highly expressed in parts of the brain that govern energy balance [41・•] and feeding behavior [42, 43]. MC4R (melanocortin 4 receptor) is reported to be responsible for up to $6 \%$ of early-onset or severe adult obesity cases [44]. In addition, disrupted $M C 4 R$ in mice causes hyperphagia, hyperinsulinemia, and hyperglycemia [45]. SH2B1 (Src-homology-2 [SH2] domain-containing putative adaptor protein-1) is associated with increased serum leptin [46]. BDNF (brain-derived neurotrophic factor) and $N R X N 3$ (neurexin 3 ) are linked to substance abuse and reward behavior, probably interfering with dopamine neurotransmission in pathways involved in reward effects, motivation, and decision making [47, 48]. $N E G R 1$ (neuronal growth regulator 1) is a growth promoter that participates in the regulation of axon outgrowth in the developing brain [37••]. TMEM18 (transmembrane protein 18) enhances the migration capacity of cells [49] and is highly expressed in the hypothalamus [36••].

Another eight genes associated with obesity are highly expressed in the hypothalamus [36••]: KCTD15 (potassium channel tetramerization domain-containing 15), GNPDA2 (glucosamine-6-phosphate deaminase-2) $\mathrm{MTCH} 2$ (mitochondrial carrier homolog 2), SDCCAG8 (serologically defined colon cancer antigen 8), FAIM2 (Fas apoptotic inhibitory molecule 2), ETV5 (ets variant 5), NCP1 (endosomal/lysosomal Niemann-Pick $\mathrm{C} 1$ gene), and $P R L$ (prolactin) are thought to be involved in obesity susceptibility via CNS-mediated effects.

Other associations observed in GWAS, which affect susceptibility to obesity, may operate peripherally (those primarily associated with fat-distribution and/or central obesity). Among these, we include the following: TFAP $2 B$ (transcription factor activating enhancer-binding protein 2 $\beta)$ preferentially expressed in adipose tissue, which is involved in glucose transport, lipid accumulation, and adiponectin expression [17••]; NCR3 (natural cytotoxicity triggering receptor 3 ) and PTER (phosphotriesterase related), which might mediate their effect through the hypothesized low-grade inflammation of adipose tissue [50]; and lastly $c-M A F$, a transcription factor involved in adipogenesis $[38 \bullet \bullet]$. In addition, the interesting female-only association to LYPLAL1 (lysophospholipase-like-1) is caused by a lipase with increased expression in subcutaneous adipose tissue [17••]. This finding is of particular interest as it supports previous hypotheses that there are sex-specific genes contributing to variation of obesity-related traits and that genes account for more variance of fat distribution in women than in men $[51 \bullet \cdot]$. It is also worth noting that although

Table 1 Details of genetic association studies

\begin{tabular}{|c|c|c|c|}
\hline Study & Study name (if any) & Number of samples in discovery cohort & Ancestry of discovery cohort \\
\hline Frayling et al. [29••] & WTCCC & 1924 & Europeans \\
\hline Scuteri et al. [30] & Sardinia & 4741 & Europeans \\
\hline Chambers et al. $[35 \bullet \bullet]$ & LOLIPOP & 2684 & Indian Asians \\
\hline Loos et al. [40] & & 16,876 & Northern European \\
\hline Heard-Costa et al. [48] & The CHARGE consortium & 31,373 & Europeans \\
\hline Lindgren et al. $[17 \bullet \bullet]$ & The GIANT consortium & 38,580 & Europeans \\
\hline Cotsapas et al. [71] & & 775 cases and 3197 unascertained controls & Europeans \\
\hline Meyre et al. $[38 \bullet \bullet]$ & & 1380 and 1416 age-matched normal-weight controls & Europeans \\
\hline Thorleifsson et al. $[37 \bullet \bullet]$ & deCODE & 37,347 & Europeans and African Americans \\
\hline Willer et al. $[36 \bullet \bullet]$ & The GIANT consortium & 32,387 & Europeans \\
\hline Hinney et al. [72] & & 487 extremely obese young cases and 442 healthy lean controls & Europeans \\
\hline Scherag et al. [39] & & 453 extremely obese young cases and 435 healthy lean controls & Europeans \\
\hline Cho et al. $[53 \bullet \bullet]$ & KARE & 8842 & Asian \\
\hline
\end{tabular}

CHARGE cohorts for heart and aging research in genomic epidemiology, GIANT genetic investigation of anthropometric traits, KARE korean association resource, LOLIPOP London life sciences prospective population, WTCCC wellcome trust case control consortium 


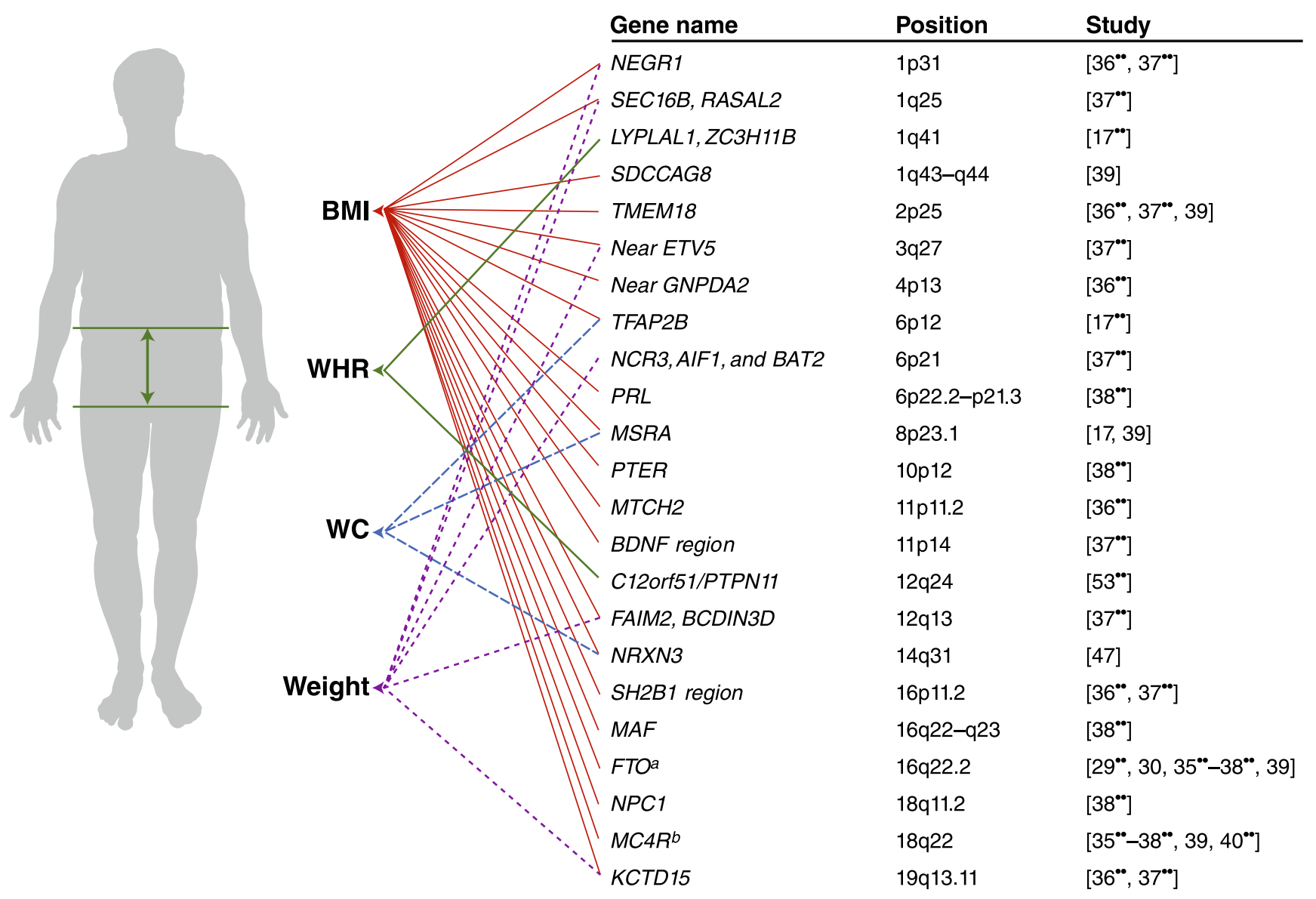

Fig. 1 Genes associated with obesity-related anthropometric measures. $B M I$ body mass index, $W C$ waist circumference, $W H R$ waist to hip ratio. ${ }^{\mathrm{a}}$ Indicates type 2 diabetes association. ${ }^{\mathrm{b}}$ Indicates association with monogenic obesity

involved in the production of leptin and thus proposed to operate through CNS, SHB2B1 also has an effect on total fat [52] and may have a dual role in obesity susceptibility.

These first insights, gained through the results of the initial wave of GWAS, support the biology that monogenic disorders point to CNS regulation of overall obesity (BMI), whereas more peripheral effects operate on central obesity and fat distribution. However, it is noteworthy that these conclusions are based on likely candidate genes in associated regions, but many of the genes are still uncharacterized and can therefore not be disregarded as candidates. Furthermore, some of the significant associations are located in gene deserts and in non-coding regions. Dissecting these associations, identifying causal variation, and unravelling the functional role is a major challenge that lies ahead.

\section{The Future of Genetic Studies in Obesity}

Despite the initial success of the GWAS strategy, the established loci together explain less than $2 \%$ of the interindividual BMI variation [17•• ] and less than $1 \%$ of the interindividual WHR variation $[36 \bullet \bullet]$. With heritability estimates of $40 \%$ to $70 \%$ for BMI and $30 \%$ to $60 \%$ for WHR (even after adjusting for BMI) [26], there must be many more susceptibility loci to uncover. Different strategies for how to do this have been proposed and are currently being explored.

Improving and Expanding GWA Studies

One approach is to continue the successful meta-analysis efforts thus far and increase the sample size and power. Such efforts are well advanced within the GIANT (Genetic Investigation of Anthropometric Traits) consortium, in which both BMI and WHR are being currently analyzed in more than 100,000 samples. This will add new common variants, with small effect sizes, that are robustly associated with these obesity phenotypes. Also, power to identify novel loci might vary between different populations, as a result of differences in allele frequencies, and because the majority of studies have been done in samples of European ancestry, power could be increased by using samples from different ancestries. One example of this is the WHR association to a locus on chromosome 12q24 (Fig. 1), reported recently in a Korean population-based cohort (Table 1) [53••]. This association is 
not possible to evaluate in current GWAS efforts in European samples because the variants are not present in the CEU samples from HapMap (and no imputation can be performed). Also, fewer samples were needed to identify the $M C 4 R$ BMI locus in Indian Asian samples than in Europeans, as the risk allele frequency is higher in Indian Asians. Therefore, expanding efforts to studies using samples of other ethnicities should provide excellent opportunities to discover additional obesity susceptibility loci.

Improving Subject Selection and the Phenotype

Most studies hereto have focused on anthropometric measures of obesity (eg, BMI, WC, WHR) because these are straightforward, noninvasive, and inexpensive surrogate measures of adiposity. Although these studies have been successful, they have required very large sample sizes for identification of robust associations (Table 1). More precise measures (eg, CT scan, dual-energy x-ray absorptiometry (DXA scan) or MRI) are more expensive and time consuming to collect, but are expected to reduce the number of samples needed to identify robust associations to adiposity. It is worth noticing that considerable variation between machines, calibrations, operators, and sites will introduce noise even in these fine-tuned adiposity measures and that the most successful studies will put emphasis on both of these.

\section{Looking Beyond Common SNPs}

\section{Rare and Low Frequency Variants}

The genotyping arrays currently used for GWAS are designed to provide excellent coverage of common variants, especially when married with genotype imputation methods. Thus far, risk allele frequencies of the obesity susceptibility variants, which are identified through GWAS, are all greater than $5 \%$. Rare and low frequency variants (minor allele frequency $<5 \%$ ), however, are not well captured by these methods. It has been discussed that rare and low frequency variants would have higher penetrance and would thus explain more of the "missing heritability" than common variants have done hereto. To date, robust associations to obesity have been observed for low frequency, nonsynonymous variants in three loci-MC4R [54], prohormone convertase 1/3 (PCSK1) [55], and BDNF [56]—in samples of European ancestry, using a traditional candidate gene approach. Fuller, genome-wide evaluation of such variants is imminent with advances in high-throughput sequencing technologies and the large efforts of the 1000 Genomes project (a massive collaborative effort to carry out a deep characterization of genomic variation in over 1000 individuals derived from a number of populations worldwide). These efforts will aid in cataloguing variants of lower frequency and might lead to new genotyping arrays and imputation methods that will capture a fuller allele frequency spectrum than we have been able to until now.

\section{Copy Number Variants}

It has recently been suggested that common copy number variants (CNVs) are unlikely to contribute substantially to the genetic basis of common human diseases [57]. The extent to which CNVs might contribute to "missing heritability" of common traits and disorders is currently not clear [58]. A large fraction of human CNVs arise from common, diallelic polymorphisms [57, 59] and most of these CNVs are in linkage disequilibrium with adjacent SNPs, so their contribution to phenotypes can be assessed via these SNPs. This strategy recently proved its value when a SNP significantly associated with BMI (NEGR1, rs2815752; Fig. 1) was also found to tag a $45-\mathrm{kb}$ deletion upstream of NEGR1. The deletion is a causal candidate for the association signal, but this needs further fine-mapping and functional work to be proven $[36 \bullet \bullet]$.

Further evidence that CNVs contribute to the genetic architecture of human obesity came with the finding that large, rare chromosomal deletions on chromosome 16p11.2 are associated with severe, highly penetrant obesity $[60 \bullet$, $61 \cdot]$. The deletions span a large number of genes including $S H 2 B 1$, which is known to be involved in leptin and insulin signaling [62]. The carriers of the deletions exhibit hyperphagia and severe insulin resistance, which resemble the phenotype in rodents with the deletion of Sh2b1 [63].

As common variants at the same locus near $S H 2 B 1$ have been associated with BMI (used as a quantitative trait) in GWAS (Fig. 1; [36••, 37••]), the data also provides further evidence that we might expect to find more loci where common variants are associated with common forms of obesity as well as rare variants that are associated with severe, extreme forms of obesity. Thus, further evaluations of the full range of CNVs are needed to fully estimate their impact on obesity.

\section{Non-Coding RNA}

Even more of the heritability of obesity might be explained by determining the role of non-coding RNAs, such as microRNAs (miRNA). These are short, non-coding RNA molecules that regulate gene expression post-transcriptionally, by binding to the $3^{\prime}$ UTR of their target genes and limiting translation into protein. Each miRNA is suspected to target several genes. miRNAs are known regulators of adipocyte differentiation, obesity, insulin resistance, and appetite regulation [64]. Thus, dysregulation of miRNA expression (caused by genetic or environmental "drivers") can potentially influence obesity susceptibility. A better 
understanding of miRNA physiology could be exploited, to elucidate the etiology of the disease, and manipulated to reduce obesity, as has already been done in animal studies for microRNA-122 [65].

\section{Epigenetic Modifications}

Modifications that affect gene expression but do not alter the DNA sequence are termed epigenetic modifications [66]. These include methylation and histone modifications, which are likely to have key roles in the inheritance and susceptibility to obesity [67], by affecting the expression of associated genes. Also, intrauterine environment during specific developmental stages can alter the epigenetic profile of an individual and may serve as a primer for the onset of obesity and other phenotypes in later age [68]. Next-generation sequencing and ChIP-Seq technology can be applied to discern the epigenetic profile of the genes already associated with obesity and may also be applied in a genome-wide approach and allow integration with existing GWAS and genome sequencing data.

\section{Differences between the metabolically deleterious and the metabolically healthy forms of obesity}

Although the majority of individuals with overall obesity develop metabolic complications, a proportion $(\sim 10 \%$ to $25 \%$ ) remains metabolically healthy [69]. It has been speculated that this is due to retained insulin sensitivity, despite the metabolic challenges that overall obesity presents. Importantly, recent, robust genetic associations with obesity phenotypes can thus be used to explore the differences between the metabolically deleterious and the metabolically healthy forms of obesity, which might aid in the distinction between obesity loci that lead to metabolic dysregulation and those that do not [70].

\section{Identifying the Causal Variant/Gene}

After identifying susceptibility loci, caution in the interpretation of the results and associations is still necessary because associated genetic variants are not always within known genes and associations can sometimes span large areas containing a number of genes. Thus, in most cases, we cannot say for certain that we have identified the "smoking gun." Large-scale fine-mapping and resequencing efforts are needed to catalogue and evaluate the genetic variation from the full allele spectra in associated regions. The use of different linkage disequilibrium patterns in samples from different ethnicities in the previously mentioned efforts is an obvious strategy to attempt to hone in on regions with higher likelihood of containing the etiologic variant. Dissecting function and proving causation of genetic variants is not necessarily a straightforward task, even for relatively simple cases of monogenic diseases, and a challenging task lies before the research community to, in a robust manner, translate these findings into characterization of function and consequences on physiology.

\section{Conclusions}

The current obesity epidemic does not have a purely genetic basis, although genetics do play a large role in susceptibility. Changes to lifestyle over the past century have created an "obesogenic environment" in which underlying individual genetic factors contributing to risk can be exposed. With the advent of GWAS, we have finally started to detect robust associations between common genetic variants and obesity. Many of the earliest genetic associations hinted that susceptibility to obesity might act through CNS action, and that the response to obesogenic environment exposure may be neurobehaviorally driven. In addition, other evidence suggests that some genetic variants act peripherally (eg, in adipose tissue).

Although GWAs have been successful in identifying obesity loci, these only explain a small fraction of the interindividual variation, so that additional genetic factors remain to be detected. However, these findings will only yield useful therapeutic interventions, once functional variants are exposed and further molecular and physiologic characterization of the genes and pathways involved is performed.

Acknowledgments Dr. Cecilia M. Lindgren is a Wellcome Trust Research Career Development Fellow (086596/Z/08/Z). The authors would like to thanks Miss Sarah L. Keildson for help with manuscript preparation.

Disclosure No potential conflicts of interest relevant to this article were reported.

Open Access This article is distributed under the terms of the Creative Commons Attribution Noncommercial License which permits any noncommercial use, distribution, and reproduction in any medium, provided the original author(s) and source are credited.

\section{References}

Papers of particular interest, published recently, have been highlighted as:

- Of importance

•- Of major importance

1. O'Rahilly S: Human genetics illuminates the paths to metabolic disease. Nature 2009, 462:307-314. 
2. Flegal KM, Graubard BI, Williamson DF, Gail MH: Causespecific excess deaths associated with underweight, overweight, and obesity. JAMA 2007, 298:2028-2037.

3. Pischon T, Boeing H, Hoffmann K, et al.: General and abdominal adiposity and risk of death in Europe. N Engl J Med 2008, 359:2105-2120.

4. Fontaine KR, Redden DT, Wang C, et al.: Years of life lost due to obesity. JAMA 2003, 289:187-193.

5. Teucher B, Rohrmann S, Kaaks R: Obesity: focus on all-cause mortality and cancer. Maturitas 65:112-116.

6. World Health Organization: Obesity: preventing and managing the global epidemic: report of a WHO Consultation on Obesity, Geneva, 3-5 June, 1997. Geneva: World Health Organization; 1998.

7. Ogden CL, Yanovski SZ, Carroll MD, Flegal KM: The epidemiology of obesity. Gastroenterology 2007, 132:2087-2102.

8. The Health and Social Care Information Centre: Statistics on obesity, physical activity and diet, England 2006. Available at http://www.ic.nhs.uk/statistics-and-data-collections/health-andlifestyles/obesity/statistics-on-obesity-physical-activity-and-dietengland-2006. Accessed September 2010.

9. Berghofer A, Pischon T, Reinhold T, et al.: Obesity prevalence from a European perspective: a systematic review. BMC Public Health 2008, 8:200.

10. Cuevas A, Alvarez V, Olivos C: The emerging obesity problem in Latin America. Expert Rev Cardiovasc Ther 2009, 7:281-288.

11. Jia WP, Wang C, Jiang S, Pan JM: Characteristics of obesity and its related disorders in China. Biomed Environ Sci 2010, 23:4-11.

12. Sniderman AD, Bhopal $\mathrm{R}$, Prabhakaran $\mathrm{D}$, et al.: Why might South Asians be so susceptible to central obesity and its atherogenic consequences? The adipose tissue overflow hypothesis. Int J Epidemiol 2007, 36:220-225.

13. Coll AP, Farooqi IS, Challis BG, et al.: Proopiomelanocortin and energy balance: insights from human and murine genetics. J Clin Endocrinol Metab 2004, 89:2557-2562.

14. Shapira NA, Lessig MC, He AG, et al.: Satiety dysfunction in Prader-Willi syndrome demonstrated by fMRI. J Neurol Neurosurg Psychiatry 2005, 76:260-262.

15. Rankinen T, Zuberi A, Chagnon YC, et al.: The human obesity gene map: the 2005 update. Obesity (Silver Spring) 2006, 14:529644.

16. Wiklund $\mathrm{P}$, Toss $\mathrm{F}$, Weinehall $\mathrm{L}$, et al.: Abdominal and gynoid fat mass are associated with cardiovascular risk factors in men and women. J Clin Endocrinol Metab 2008, 93:4360-4366.

17. • Lindgren CM, Heid IM, Randall JC, et al.: Genome-wide association scan meta-analysis identifies three Loci influencing adiposity and fat distribution. PLoS Genet 2009, 5:e1000508. This is a large collaborative meta-analysis that identified the first robustly WHR-associated locus, which shows a strong sexspecific association in women.

18. Poulsen P, Vaag A, Kyvik K, Beck-Nielsen H: Genetic versus environmental aetiology of the metabolic syndrome among male and female twins. Diabetologia 2001, 44:537-543.

19. Stunkard AJ, Foch TT, Hrubec Z: A twin study of human obesity. JAMA 1986, 256:51-54.

20. Turula M, Kaprio J, Rissanen A, Koskenvuo M: Body weight in the Finnish Twin Cohort. Diabetes Res Clin Pract 1990, 10(Suppl 1):S33-S36.

21. Wardle J, Carnell S, Haworth CM, Plomin R: Evidence for a strong genetic influence on childhood adiposity despite the force of the obesogenic environment. Am J Clin Nutr 2008, 87:398-404.

22. Stunkard AJ, Sorensen TI, Hanis C, et al.: An adoption study of human obesity. N Engl J Med 1986, 314:193-198.

23. Moll PP, Burns TL, Lauer RM: The genetic and environmental sources of body mass index variability: the Muscatine Ponderosity Family Study. Am J Hum Genet 1991, 49:1243-1255.
24. Magnusson PK, Rasmussen F: Familial resemblance of body mass index and familial risk of high and low body mass index. A study of young men in Sweden. Int J Obes Relat Metab Disord 2002, 26:1225-1231.

25. Selby JV, Newman B, Quesenberry CP Jr, et al.: Evidence of genetic influence on central body fat in middle-aged twins. Hum Biol 1989, 61:179-194.

26. Rose KM, Newman B, Mayer-Davis EJ, Selby JV: Genetic and behavioral determinants of waist-hip ratio and waist circumference in women twins. Obes Res 1998, 6:383-392.

27. Eaton SB, Konner M, Shostak M: Stone agers in the fast lane: chronic degenerative diseases in evolutionary perspective. Am J Med 1988, 84:739-749.

28. Saunders CL, Chiodini BD, Sham P, et al.: Meta-analysis of genome-wide linkage studies in BMI and obesity. Obesity (Silver Spring) 2007, 15:2263-2275.

29. ••Frayling TM, Timpson NJ, Weedon MN, et al.: A common variant in the FTO gene is associated with body mass index and predisposes to childhood and adult obesity. Science 2007, 316:889-894. This is the first genome-wide search for type 2 diabetes susceptibility genes that identified an unequivocal BMI association to a common variant in the FTO gene.

30. Scuteri A, Sanna S, Chen WM, et al.: Genome-wide association scan shows genetic variants in the FTO gene are associated with obesity-related traits. PLoS Genet 2007, 3:e115.

31. International HapMap Consortium: A haplotype map of the human genome. Nature 2005, 437:1299-1320.

32. National Center for Biotechnology Information, National Library of Medicine: Database of Single Nucleotide PolymorphismsdbSNP Build ID: 131. Available at http://www.ncbi.nlm.nih.gov/ SNP/. Accessed September 2010.

33. Barrett JC, Cardon LR: Evaluating coverage of genome-wide association studies. Nat Genet 2006, 38:659-662.

34. Pe'er I, de Bakker PI, Maller J, et al.: Evaluating and improving power in whole-genome association studies using fixed marker sets. Nat Genet 2006, 38:663-667.

35. ${ }^{\circ}$ Chambers JC, Elliott P, Zabaneh D, et al.: Common genetic variation near MC4R is associated with waist circumference and insulin resistance. Nat Genet 2008, 40:716-718. This is the first large-scale screen in non-European (Indian Asians) samples for obesity genes that identifies and/or replicates both FTO and $M C 4 R$ to be associated with measures of obesity and insulin resistance.

36. • Willer CJ, Speliotes EK, Loos RJ, et al.: Six new loci associated with body mass index highlight a neuronal influence on body weight regulation. Nat Genet 2009, 41:25-34. This is a large-scale search for genes associated with common obesity as measured by BMI that yielded six novel, robust obesity susceptibility loci. A number of the genes in the associated loci are expressed in and/or are known to act in the hypothalamus.

37. •-Thorleifsson G, Walters GB, Gudbjartsson DF, et al.: Genome-wide association yields new sequence variants at seven loci that associate with measures of obesity. Nat Genet $2009,41: 18-24$. This was a search for common variants that affect two measures of obesity - weight and BMI-in European and African samples that identified seven novel, significantly associated obesity loci.

38. • Meyre D, Delplanque J, Chevre JC, et al:: Genome-wide association study for early-onset and morbid adult obesity identifies three new risk loci in European populations. Nat Genet 2009, 41:157-159. This was the first large-scale GWAS for extreme forms of obesity (early-onset and morbid adult obesity) that identifies three novel obesity loci showing little overlap with the GWAS for quantitative measures of obesity.

39. Scherag A, Dina C, Hinney A, et al.: Two new Loci for bodyweight regulation identified in a joint analysis of genome-wide 
association studies for early-onset extreme obesity in French and German study groups. PLoS Genet 2010, 6:e1000916.

40. • Loos RJ, Lindgren CM, Li S, et al.: Common variants near MC4R are associated with fat mass, weight and risk of obesity. Nat Genet 2008, 40:768-775. After combining GWA data from 16,876 individuals, a locus about $188-\mathrm{kb}$ downstream of the $M C 4 R$ gene was associated with BMI. MC4R mutations are the leading cause of monogenic severe childhood-onset obesity.

41. •Gerken T, Girard CA, Tung YC, et al.: The obesity-associated FTO gene encodes a 2-oxoglutarate-dependent nucleic acid demethylase. Science 2007, 318:1469-1472. This is a functional study in which both bioinformatics work and animal models point to FTO having DNA methylation activity, with expression regulated by energy intake patterns.

42. Fredriksson R, Hagglund M, Olszewski PK, et al.: The obesity gene, FTO, is of ancient origin, up-regulated during food deprivation and expressed in neurons of feeding-related nuclei of the brain. Endocrinology 2008, 149:2062-2071.

43. Wardle J, Llewellyn C, Sanderson S, Plomin R: The FTO gene and measured food intake in children. Int J Obes (Lond) 2009, 33:42-45.

44. O'Rahilly S, Farooqi IS: Human obesity: a heritable neurobehavioral disorder that is highly sensitive to environmental conditions. Diabetes 2008, 57:2905-2910.

45. Huszar D, Lynch CA, Fairchild-Huntress V, et al.: Targeted disruption of the melanocortin-4 receptor results in obesity in mice. Cell 1997, 88:131-141.

46. Li Z, Zhou Y, Carter-Su C, et al.: SH2B1 enhances leptin signaling by both Janus kinase 2 Tyr813 phosphorylationdependent and -independent mechanisms. Mol Endocrinol 2007, 21:2270-2281.

47. Gratacos M, Gonzalez JR, Mercader JM, et al.: Brain-derived neurotrophic factor Val66Met and psychiatric disorders: metaanalysis of case-control studies confirm association to substancerelated disorders, eating disorders, and schizophrenia. Biol Psychiatry 2007, 61:911-922.

48. Heard-Costa NL, Zillikens MC, Monda KL, et al.: NRXN3 is a novel locus for waist circumference: a genome-wide association study from the CHARGE Consortium. PLoS Genet 2009, 5:e1000539.

49. Jurvansuu J, Zhao Y, Leung DS, et al.: Transmembrane protein 18 enhances the tropism of neural stem cells for glioma cells. Cancer Res 2008, 68:4614-4622.

50. Virtue S, Vidal-Puig A: It's not how fat you are, it's what you do with it that counts. PLoS Biol 2008, 6:e237.

51. •ZZillikens MC, Yazdanpanah M, Pardo LM, et al.: Sex-specific genetic effects influence variation in body composition. Diabetologia 2008, 51:2233-2241. This study provides evidence for sexspecific genetic effects that may underlie sex differences for several body composition and fat distribution traits.

52. Jamshidi Y, Snieder H, Ge D, et al.: The SH2B gene is associated with serum leptin and body fat in normal female twins. Obesity (Silver Spring) 2007, 15:5-9.

53. ${ }^{\circ}$ Cho YS, Go MJ, Kim YJ, et al.: A large-scale genome-wide association study of Asian populations uncovers genetic factors influencing eight quantitative traits. Nat Genet 2009, 41:527-534. This is the first Korean GWAS that identifies a WHR association on chromosome $12 \mathrm{q} 24$, to a variant that is not in HapMap and thus has not been evaluated in European populations yet.

54. Stutzmann F, Vatin V, Cauchi S, et al.: Non-synonymous polymorphisms in melanocortin-4 receptor protect against obesity: the two facets of a Janus obesity gene. Hum Mol Genet 2007, 16:1837-1844.

55. Benzinou M, Creemers JW, Choquet H, et al.: Common nonsynonymous variants in PCSK1 confer risk of obesity. Nat Genet 2008, 40:943-945.

56. Shugart YY, Chen L, Day IN, et al.: Two British women studies replicated the association between the Val66Met polymorphism in the brain-derived neurotrophic factor (BDNF) and BMI. Eur J Hum Genet 2009, 17:1050-1055.

57. Conrad DF, Pinto D, Redon R, et al.: Origins and functional impact of copy number variation in the human genome. Nature 2010, 464:704-712.

58. Manolio TA, Collins FS, Cox NJ, et al.: Finding the missing heritability of complex diseases. Nature 2009, 461:747-753.

59. McCarroll SA, Kuruvilla FG, Korn JM, et al.: Integrated detection and population-genetic analysis of SNPs and copy number variation. Nat Genet 2008, 40:1166-1174.

60. $\bullet$ Walters RG, Jacquemont S, Valsesia A, et al.: A new highly penetrant form of obesity due to deletions on chromosome 16p11.2. Nature 2010, 463:671-675. This group identified deletions on chromosome $16 \mathrm{p} 11.2$ to be associated with morbid, adult forms of obesity.

61. •Bochukova EG, Huang N, Keogh J, et al.: Large, rare chromosomal deletions associated with severe early-onset obesity. Nature 2010, 463:666-670. Here large, rare deletions on chromosome $16 \mathrm{p} 11.2$ are shown to be associated with severe early-onset obesity. The deletion overlaps with a locus that is associated with BMI and contains several genes including $S H 2 B 1$.

62. Farooqi IS, Wangensteen $\mathrm{T}$, Collins $\mathrm{S}$, et al.: Clinical and molecular genetic spectrum of congenital deficiency of the leptin receptor. N Engl J Med 2007, 356:237-247.

63. Morris DL, Cho KW, Zhou Y, Rui L: SH2B1 enhances insulin sensitivity by both stimulating the insulin receptor and inhibiting tyrosine dephosphorylation of insulin receptor substrate proteins. Diabetes 2009, 58:2039-2047.

64. Krutzfeldt J, Stoffel M: MicroRNAs: a new class of regulatory genes affecting metabolism. Cell Metab 2006, 4:9-12.

65. Xie H, Sun L, Lodish HF: Targeting microRNAs in obesity. Expert Opin Ther Targets 2009, 13:1227-1238.

66. Bird A: Perceptions of epigenetics. Nature 2007, 447:396-398.

67. Stoger R: Epigenetics and obesity. Pharmacogenomics 2008, 9:1851-1860.

68. Waterland RA, Jirtle RL: Early nutrition, epigenetic changes at transposons and imprinted genes, and enhanced susceptibility to adult chronic diseases. Nutrition 2004, 20:63-68.

69. Bluher $\mathrm{M}$ : The distinction of metabolically 'healthy' from 'unhealthy' obese individuals. Curr Opin Lipidol 2010, 21:3843.

70. Freathy RM, Timpson NJ, Lawlor DA, et al.: Common variation in the FTO gene alters diabetes-related metabolic traits to the extent expected given its effect on BMI. Diabetes 2008, 57:14191426.

71. Cotsapas C, Speliotes EK, Hatoum IJ, et al.: Common body mass index-associated variants confer risk of extreme obesity. Hum Mol Genet 2009, 18:3502-3507.

72. Hinney A, Nguyen TT, Scherag A, et al.: Genome wide association (GWA) study for early onset extreme obesity supports the role of fat mass and obesity associated gene (FTO) variants. PLoS One 2007, 2:e1361. 